\title{
Qualitätszirkel für Praxisassistentinnen - ein Schritt in die Zukunft der Grundversorgung
}

\author{
Zeitintensive Aufgaben im Praxismanagement, in der Beratung, Schulung und Motiva- \\ tion vor allem von Langzeitpatienten können viele Ärzte kaum bewältigen. Entlastung \\ kann von den Medizinischen Praxisassistentinnen kommen. Dafür bedarf es aber einer \\ Weiterentwicklung des Berufsbildes im Sinne einer selbständigen Mitarbeiterin der \\ Grundversorger. MPA-Qualitätszirkel können dazu einen wichtigen Beitrag leisten.
}

\section{Hansueli Schläpfera, \\ Valeria Maissen ${ }^{b}$, \\ Beatrice Birnbaumc, \\ Marco Zollerd}

Mitglieder der AG Qualitätszirkel für MPA

a Vertreter medswiss.net

b Praxismanagement mediX zürich

c Erwachsenenbildnerin, Vertreterin SVA

d Dr. med.,

Vertreter Hausärzte Schweiz

Wir danken dem Schweizerischen Verband Medizinischer Praxisassistentinnen SVA, Hausärzte Schweiz und medswiss.net für die Unterstützung in der Trägerschaft der Kurse.
Korrespondenz:

Dr. med. Hansueli Schläpfer Innere Medizin FMH Leiter Ärztenetz säntiMed Kasernenstrasse 30

CH-9100 Herisau

Tel. 0713522666

Fax 0713524256

schlaepfer11@bluewin.ch

\section{Krise der Grundversorgung}

Wir alle wissen: Die medizinische Grundversorgung ist unabdingbar für ein gut funktionierendes Gesundheitswesen. Trotzdem geht es ihr nicht gut: Überlastete Hausärzte und -ärztinnen, mangelnder Nachwuchs, Prestige- und Einkommensverluste verstärken sich gegenseitig zum Teufelskreis. Erste Gegenmassnahmen wurden ergriffen, aber es wird lange dauern, bis sie zur Wirkung kommen. In der Zwischenzeit gilt es, das zu tun, was in unserer Macht liegt: Aktivieren wir das Potential der Medizinischen Praxisassistentinnen (MPA)!

\section{Neue Rolle für die MPA}

Die heutige MPA war ursprünglich Dienstmädchen «für alles» im Arzthaushalt. Aus ihr wurde die Arztgehilfin, heute die Praxisassistentin und in Zukunft wird es die Mitarbeiterin des Arztes sein. Die rasch zunehmenden, zeitintensiven Aufgaben im Praxismanagement, in der Beratung, Schulung und Motivation vor allem bei Langzeitpatienten können von den Ärzten schon jetzt kaum mehr bewältigt werden [1]. Doch: Müssen wirklich alles die Ärzte übernehmen? Sehr oft handelt es sich um Routinesituationen, die keine ärztliche Kompetenz erfordern. Sie können sehr gut (und manchmal sogar besser) von entsprechend geschulten MPAs selbständig ausgeführt werden. Dies verlangt allerdings eigenständiges und kritisches Denken bei den MPAs und ein Umdenken bei den Ärzten.

\section{Qualitätszirkel - Weg in die Selbständigkeit} Qualitätszirkel sind bei den Ärzten und namentlich bei den Grundversorgern längst bewährt. Sie sind ein wichtiges Instrument zur Emanzipation der Grundversorger vom Definitions-Monopol der Professoren, Konsensuskonferenzen und der Industrie. Hier wird der kritische Geist geschult, werden die wirklichen Praxisprobleme formuliert und realitätsgerechte Antworten gefunden. Warum also sollten sie nicht auf die MPAs übertragen werden, wenn sie in Zukunft ähnliche Fähigkeiten brauchen?

\section{Cercles de qualité pour assistantes médicales - un pas vers I'avenir de la médecine de premier recours}

A l'avenir, les tâches dévolues à la médecine de premier recours ne pourront être accomplies et mâ̂trisées par les cabinets médicaux que s'ils utilisent davantage et de manière ciblée le potentiel des assistantes médicales. Cela suppose une évolution de cette profession en vue de concéder plus d'autonomie aux collaboratrices pour seconder le médecin. Les réseaux de médecins ont été les premiers à reconnaître que les cercles de qualité, tels qu'ils se sont établis pour les médecins mais cette fois à l'intention des assistantes médicales, représentent un moyen de faire évoluer cette profession. Un groupe de travail composé de représentants de l'Association suisse des assistantes médicales (ASA), de Médecins de famille Suisse et de medswiss.net a été mis sur pied pour former les responsables nécessaires à ces cercles de qualité et proposer des cours.

MPAs haben gelernt, ärztliche Verordnungen genau (und oft unkritisch) zu befolgen. So wichtig dies ist, es hemmt die Selbständigkeit im Denken und Arbeiten, die heute für die Weiterentwicklung der Grundversorgung unentbehrlich sind. Genau hier setzt der Qualitätszirkel für MPAs an: Es werden Hemmungen abgebaut, die MPAs lernen, eigene Themen $\mathrm{zu}$ formulieren, Erfahrungen auszutauschen und neue Lösungen zu suchen. Dies kann bis zur Erarbeitung eigener Merkblätter und «MPA-Guidelines» führen. 
Die Qualitätszirkel müssen natürlich organisiert und moderiert werden. Dies ist ein weiteres Übungsfeld, auf dem die MPAs ihre oft brachliegenden Fähigkeiten entfalten und dann auch in den Praxen nutzbringend anwenden können. Besonders in den immer zahlreicher werdenden Gruppenpraxen sind diese Qualitäten gefragt.

\section{Es braucht Leiterinnen für Qualitätszirkel}

Die Leitungsfunktion ist für die MPAs meist ungewohnt, für einen erfolgreichen Qualitätszirkel aber sehr wichtig. Eine Ausbildung zur Leiterin eines Qualitätszirkels (QZ) ist deshalb von grosser Bedeutung und gibt der MPA die notwendige Sicherheit. In einem Pilotversuch organisierte deshalb mediX schweiz in Zusammenarbeit mit der Schweizerischen Gesellschaft für Allgemeinmedizin SGAM (Arbeitsgruppe Moderatorenkurse) in den Jahren 2009/2010 drei solcher Moderatorinnenkurse mit insgesamt 35 Teilnehmerinnen. Vorbild waren die Kurse der SGAM, und es war sehr wertvoll, die erfahrenen Ausbildner der SGAM für diese Kurse in Anspruch nehmen zu können.

Rasch zeigte sich, dass für Inhalt und Organisation dieser Kurse eine strukturierte Trägerschaft unerlässlich ist. Erfreulicherweise gelang es, den Schweizerischen Verband Medizinischer Praxisassistentinnen (SVA), Hausärzte Schweiz und medswiss.net (Schweizer

\section{«Ärztenetze waren und sind die wichtigsten Promotoren für die Entstehung solcher MPA- Qualitätszirkel.»}

Dachverband der Ärztenetze) als Träger zu gewinnen. Sie finanzieren die Ausarbeitung eines eigenen Konzepts mit den nötigen Schulungsunterlagen und organisieren in Zukunft die Moderatorinnenkurse (s. unten). Diese innovative Zusammenarbeit spiegelt die neue Teambildung von Ärztenetzen-ÄrztenMPAs, die für eine integrierte Grundversorgung notwendig ist.

\section{Die Rolle der Ärztenetze}

Ärztenetze waren und sind die wichtigsten Promotoren für die Entstehung solcher MPA-Qualitätszirkel. Ihr Interesse liegt vor allem in der konkreten und einheitlichen Umsetzung von Aufgaben und Projekten, welche die Netzpraxen leisten müssen: Überweisungsmanagement, Qualitätsnachweis, Hygiene- und Entsorgungskonzepte, einfache Patientenberatungen usw. Dazu kommt die generelle Förderung des Qualitätsbewusstseins (CIRS usw.) und der kommunikati-

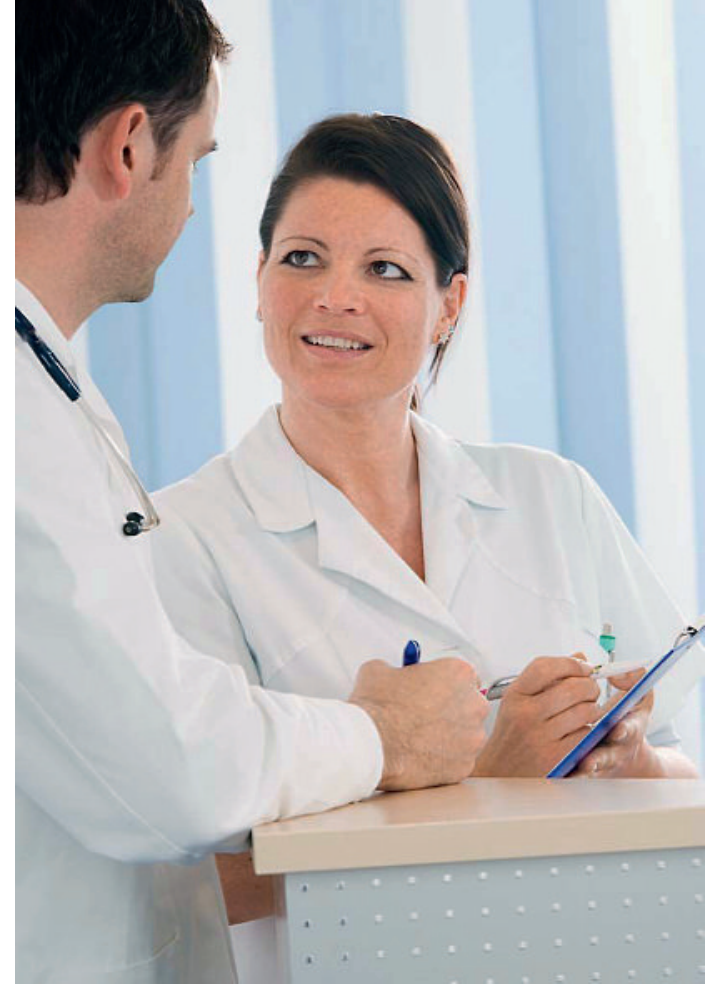

Wird die Medizinische Praxisassistentin in Zukunft die Ärzte noch mehr entlasten können?

ven Fähigkeiten der MPAs. Netze sind deshalb in der Regel bereit, Moderatorinnen auszubilden und Qualitätszirkel zu finanzieren. Sie fördern auch den Transfer von Resultaten in den Praxisalltag und möchten im Gegenzug bei der Themenwahl einen Einfluss ausüben. Auf jeden Fall ist ein Delegierter der Netzleitung wichtig, um den Austausch mit dem MPA-Qualitätszirkel zu gewährleisten.

Dies bedeutet natürlich nicht, dass es MPA-Qualitätszirkel nur in Ärztenetzen geben soll. Wenn jemand die Initiative ergreift, ist dies auch in einem Notfallkreis oder anderen Praxis-Gruppierungen möglich und sinnvoll. Ebenso ist es denkbar, dass in Zukunft auch Qualitätszirkel von Praxisassistentinnen aus Spezialarztpraxen gebildet werden.

Die Entwicklung steht erst am Anfang, und die Rückmeldungen aus den beteiligten Praxen sind fast ausnahmslos positiv. Es lohnt sich für Netze, Praxen und MPAs, MPA-Qualitätszirkel zu initiieren und damit Erfahrungen zu sammeln. Der nächste Moderatorinnen-Kurs findet am 23./24. September 2011 statt (s. www.sva.ch/bildung/fortbildung.html).

\section{Literatur}

1 Rosenmann T, Schalch E, Birnbaum B, Zanoni U. Grundversorgung: Der Weg aus der Krise führt auch über die MPA. Schweiz Ärztezeitung. 2010;91(28/29):1081-3. 